\title{
PARTICIÓN DE BIOMASA AÉREA EN PROCEDENCIAS DE Pinus greggii PLANTADAS EN EL SUR DE MÉXICO
}

\section{ABOVEGROUND BIOMASS ALLOCATION OF Pinus greggii PROVENANCES PLANTED IN SOUTHERN MÉXICO}

\author{
Daniel E. Villegas-Jiménez ${ }^{1}$, Gerardo Rodríguez-Ortiz ${ }^{1 \star}$, Vicente A. Velasco-Velasco', \\ Judith Ruiz-Luna ${ }^{1}$, José C. Carrillo-Rodríguez ${ }^{1}$ y Susana E. Ramírez-Sánchez ${ }^{2}$
}

\begin{abstract}
${ }^{1}$ Instituto Tecnológico del Valle de Oaxaca. Ex Hacienda de Nazareno. 71230, Xoxocotlán, Oaxaca. Tel. 01(951) $5170788 .{ }^{2}$ Centro Nacional de Recursos Genéticos-Instituto Nacional de Investigaciones Forestales, Agrícolas y Pecuarias, Blvd. de la Biodiversidad. 47600, Tepatitlán, Jal.

^Autor para correspondencia (grodriguez@colpos.mx, grodriguezortiz@hotmail.com)
\end{abstract}

\section{RESUMEN}

La asignación de biomasa en los órganos del árbol puede ser influenciada por factores climáticos y edafológicos, competencia lumínica, y por diferencias genéticas de los árboles. El objetivo fue estimar los componentes de biomasa aérea en 13 procedencias mexicanas de Pinus greggii Engelm. plantadas en 1997 en el sur de México. Se utilizaron datos de biomasa obtenidos en 2011 de 106 muestras aleatorias de ramas, 60 árboles medidos en pie y datos generales de la plantación establecida bajo un diseño experimental de bloques al azar. Se encontraron diferencias significativas $(P \leq 0.05)$ entre procedencias en acumulación de biomasa en acículas y madera con corteza. La mayor biomasa foliar fue para las procedencias del centro del país, El Piñón y Molango $\left(5.1 \mathrm{t} \mathrm{ha}^{-1}\right)$, mientras que la procedencia norteña, Los Lirios, acumuló la mayor cantidad de biomasa en madera con corteza $\left(19.4 \mathrm{t} \mathrm{ha}^{-1}\right)$. No hubo diferencias en biomasa de ramas cuyo promedio fue de $9.5 \mathrm{t} \mathrm{ha}^{-1}$, ni en la biomasa aérea total acumulada que fue de $25.6 \mathrm{t} \mathrm{ha}^{-1}$, en árboles de 14 años de edad para todas las procedencias. La biomasa se distribuyó 52.0, 33.6 y $\mathbf{1 4 . 4} \%$ para madera con corteza, ramas y follaje, respectivamente.

Palabras clave: Pinus greggii, acumulación de biomasa, piso forestal, procedencias.

\section{SUMMARY}

Biomass allocation on the tree may be influenced by climate, soil conditions, light competition and genetic differences among trees. Aboveground biomass components were estimated in 13 Mexican provenances of Pinus greggii Engelm. planted in 1997 in Southern México. Biomass data were collected in 2011 from 106 random samples of branches and 60 standing trees. Data sampling was distributed under a randomized block design. Significant differences $(P \leq 0.05)$ among provenances in biomass accumulation of needles and wood + bark were found. The highest leaf biomass accumulation was for provenances from Central México, El Piñón y Molango (5.1 $\left.\mathrm{t} \mathrm{ha}^{-1}\right)$. Among the northern provenances, Los Lirios accumulated the highest biomass in wood + bark $\left(19.4 \mathrm{t} \mathrm{ha}^{-1}\right)$. No differences among provenances were found in branch biomass, with an average of $9.5 \mathrm{t} \mathrm{ha}^{-1}$, and neither in total accumulated biomass which was $25.6 \mathrm{t} \mathrm{ha}^{-1}$, for 14 year-old trees. Total biomass was distributed 52.0, 33.6 and $\mathbf{1 4 . 4} \%$ for wood + bark, branches and leaves, respectively.

Index words: Pinus greggii, biomass accumulation, forest floor, provenances.

\section{INTRODUCCIÓN}

La determinación de biomasa en un bosque permite estimar los montos de materia orgánica en cada uno de sus componentes. Además, como la vegetación forestal absorbe $\mathrm{CO}_{2}$ durante su crecimiento, fija el carbono para la composición de su estructura en tallos, ramas, hojas y raíces, y libera oxígeno, también proporciona un beneficio ambiental al planeta (Pimienta et al., 2007).

Según Ferrere et al. (2008), el dióxido de carbono es un gas invernadero que contribuye al calentamiento global en una magnitud que oscila entre 50 y $75 \%$ del total de gases con efecto invernadero, cuya concentración ha aumentado de 280 ppm ( $\left.6363 \mu \mathrm{mol} \mathrm{L}^{-1}\right)$ en el año 1750 a 398 ppm (9040 $\left.\mu \mathrm{mol} \mathrm{L}{ }^{-1}\right)$ en 2013. Ante tal incremento se han implementado estrategias para la captura de carbono, y por tanto su disminución en la atmósfera, a través de mecanismos bióticos y abióticos. El primer mecanismo comprende la captura del $\mathrm{CO}_{2}$ como biomasa mediante la fotosíntesis realizada por las plantas (Díaz-Franco et al., 2007).

Entre los diferentes métodos de estimación de $\mathrm{CO}_{2}$ fijado por la vegetación, los más simples son los que involucran el contenido de carbono en proporción de la biomasa seca, que en la madera de coníferas arbóreas dicha proporción se encuentra entre 50 y $53 \%$, mientras que en especies de hoja ancha varía entre 47 y 50 \% (Rojo-Martínez et al., 2003; Delgado et al., 2005). A diferencia de las plantas anuales, los árboles almacenan los fotoasimilados en componentes de carbono en sus estructuras leñosas por periodos prolongados, por lo que se les considera reservas naturales de este elemento (Acosta-Mireles et al., 2002; Bloomberg et al., 2008). En este contexto la captura de $C$ en los sistemas forestales y agroforestales ha generado un interés internacional, debido a su potencial impacto en beneficio de la agricultura y cambio climático (Návar et al., 2004; Kaonga y Bayliss-Smith, 2009). 
Los ensayos de especies y procedencias es un procedimiento experimental empleado para comparar el comportamiento y la productividad de germoplasma provenientes de diferentes poblaciones. Estos ensayos se pueden establecer en ambientes extremos en los cuales se pretende establecer la plantación, con la finalidad de encontrar las mejores fuentes de semillas en términos de adaptación, sobrevivencia, crecimiento y productividad de los árboles (RodríguezLaguna et al., 2009). Pinus greggii ha demostrado potencial para adaptarse a lugares con poca humedad (López-Upton et al., 2004), además de presentar mayores valores de sobrevivencia, crecimiento en altura, diámetro y volumen, comparado con los pinos P. brutia, P. pseudostrobus Lindl., P. cembroides Zucc., y P. halepensis Mill. (Domínguez et al., 2001).

Además de sus características de importancia ecológica y calidad de madera, $P$. greggii es considerado con un alto potencial para el establecimiento de plantaciones forestales con fines de disminuir la presión en los bosques naturales (Morante et al., 2005). En un estudio de variación isoenzimática realizado en 10 plantaciones de Pinus greggii, incluida la procedencia Los Lirios, Coah., Ramírez et al. (1997) encontraron grandes diferencias entre poblaciones y escasa variación dentro de las poblaciones. Estas características permiten evaluar con mayor certidumbre la productividad en biomasa de esta especie bajo diferentes condiciones de sitio (Cornejo et al., 2009).

Por esta razón, el objetivo de este estudio fue estimar la partición de biomasa aérea y la biomasa acumulada en el piso forestal de 13 procedencias de $P$. greggii del norte y centro de México, plantadas en el sur del país.

\section{MATERIALES Y MÉTODOS}

\section{Área de estudio}

El ensayo se hizo en la localidad de Magdalena Zahuatlán en la región Mixteca Alta de Oaxaca, que representa la plantación de $P$. greggii más sureña del país. La altitud del sitio es de $2150 \mathrm{~m}$, cuyo clima es templado subhúmedo con lluvias en verano, temperatura media de $15^{\circ} \mathrm{C}$ y $650 \mathrm{~mm}$ de precipitación anual. El suelo es franco arcillo-arenoso, $\mathrm{pH}$ de 8.1 , materia orgánica de $2.5 \%$, nitrógeno total de $0.1 \%$, contenido de calcio de $61.13 \%$ y conductividad eléctrica de $0.14 \mathrm{dS} \mathrm{m}^{-1}$ (Valencia et al., 2006).

El material experimental incluyó 13 procedencias de $P$. greggii, seis del noroeste del país y siete del centro, que constituyeron los tratamientos. La plantación se hizo en 1997, con el sistema de plantación de cepa común (cada árbol plantado en superficie de $40 \times 40 \mathrm{~cm}$, y $40 \mathrm{~cm}$ de profundidad), con una distribución de tres bolillo (posición trian- gular) a una separación de $3 \times 3 \mathrm{~m}$, para una densidad de 1100 individuos ha ${ }^{-1}$. El diseño experimental fue de bloques completos al azar, con 12 bloques y nueve plantas por unidad experimental, para un total de 1404 plantas.

\section{Estimación de biomasa en ramas $\mathrm{y}$ acículas por rama}

A 14 años de haberse establecido la plantación (2011), se seleccionaron aleatoriamente 106 ramas de diferentes tamaños, las cuales fueron separadas de los árboles. En cada rama se midió longitud (LR, en $\mathrm{cm}$ ), diámetro basal (DBR, en $\mathrm{mm}$ ) con un vernier Scala $222 \mathrm{~A}{ }^{\circledR}$ (Gimbel Mexicana S.A. de C.V., México, D. F), área basal (ABR, en $\left.\mathrm{cm}^{2}\right)$, y altura de inserción en el fuste (AI, en m).

Las acículas se separaron de las ramas para obtener peso verde ( $\mathrm{PV}$, en $\mathrm{g}$ ) de ambos componentes con una balanza electrónica Excell ${ }^{\circledR}$ modelo BH-1200 (Barcelona, España). El material se almacenó en bolsas de papel identificadas por árbol y número de rama.

Las muestras así procesadas se trasladaron a laboratorio, donde se secaron en estufa a $70^{\circ} \mathrm{C}$ hasta peso constante. Con balanza electrónica se registró peso seco (PS, en g) de acículas y de ramas por separado.

Para estimar biomasa de rama y acículas mediante otras variables no destructivas, se ajustaron los siguientes modelos de regresión (Rodríguez-Ortiz et al., 2012):

$$
\begin{aligned}
& B A A=60.8573 \times A B R^{0.6229} \times A I^{0.0618} \ldots(\text { Ec. } 1) \\
& B A R=61.9935 \times A B R^{1.1388} \times A I^{-0.1266} \ldots \text { (Ec. 2) }
\end{aligned}
$$

donde: $\mathrm{BAA}=$ biomasa de acículas $(\mathrm{kg}), \mathrm{BAR}=$ biomasa de ramas $(\mathrm{kg}), \mathrm{ABR}=$ área basal de rama $(\mathrm{cm}), \mathrm{AI}=$ altura de inserción $(\mathrm{m})$. Las bondades de ajuste $\left(\mathrm{R}^{2}\right)$ fueron de $0.88 \mathrm{y}$ 0.89 , para las Ecs. 1 y 2 respectivamente.

\section{Estimación de biomasa de acículas y ramas a nivel árbol}

En la plantación, se seleccionaron al azar 60 árboles en pie para así tratar de muestrear toda la variabilidad existente en tamaño. En cada individuo en pie se midió altura (ALT, $\mathrm{m})$, diámetro normal $(\mathrm{DN}, \mathrm{cm})$, diámetro basal $(\mathrm{DB}$, $\mathrm{cm})$, diámetro del tallo donde inicia la copa viva $(\mathrm{DCV}, \mathrm{cm})$, diámetro de copa (DC, $\mathrm{m}$ ), longitud de fuste limpio (LFL, $\mathrm{m})$, longitud de copa (LC, $\mathrm{m}$ ) y diámetro basal de ramas (DBR, cm).

Con las Ecs. 1 y 2 se calculó la biomasa asignada a acículas y la asignada a ramas, a nivel árbol. En la muestra 
de árboles se ajustaron modelos de regresión para estimar componentes de biomasa aérea, con base en las variables no destructivas. Los modelos de mejor ajuste fueron (Rodríguez-Ortiz et al., 2012):

$$
\begin{aligned}
& B A A=0.1258 \times(D C V \times A L T)^{0.887} \quad \ldots(\text { Ec. } 3) \\
& B A R=0.0233 \times D C V^{2.8387} \times L C^{-0.6946} \quad \ldots(\text { Ec. } 4)
\end{aligned}
$$

Donde: $\mathrm{BAA}$ = biomasa acumulada en acículas por árbol $(\mathrm{kg}), \mathrm{BAR}=$ biomasa acumulada en ramas por árbol $(\mathrm{kg})$, $\mathrm{DCV}=$ diámetro de copa viva $(\mathrm{cm}), \mathrm{ALT}=$ altura total de árbol $(\mathrm{m})$ y LC = longitud de copa. Para la Ec. 3 la bondad de ajuste $\left(\mathrm{R}^{2}\right)$ fue de 0.94 , con $\mathrm{CV}=27.21 \%$, $\mathrm{CME}=0.2613$, $\mathrm{P}<0.001$; y para la Ec. 4 los valores respectivos fueron $\mathrm{R}^{2}=$ $0.92, \mathrm{CV}=42.3 \%, \mathrm{CME}=1.6388, \mathrm{P}<0.0001$.

\section{Estimación de biomasa en madera con corteza}

Cada uno de los 60 árboles se cubicó en pie, con base en medidas de diámetro con corteza $(\mathrm{cm})$ inferior y superior de cada troza de $1 \mathrm{~m}$ de longitud, hasta llegar a la punta del tallo; en algunos casos se tuvo que escalar el árbol o auxiliarse de una escalera.

El volumen verde $\left(\mathrm{VOL}, \mathrm{m}^{3}\right)$ de tallo se estimó mediante el método de trozas traslapadas (Bayle, 1995). Aleatoriamente se seleccionaron tres rodajas de $5 \mathrm{~cm}$ de grosor de cada árbol-procedencia (39 muestras), como muestra para determinar su gravedad específica (GE). En cada rodaja se registró peso verde $(\mathrm{PV}, \mathrm{g})$ y volumen verde $\left(\mathrm{VV}, \mathrm{cm}^{3}\right)$ por desplazamiento de agua en un vaso de precipitados de 500 $\mathrm{mL}$. Las muestras de madera se secaron en estufa a temperatura de $90{ }^{\circ} \mathrm{C}$ hasta peso constante, y se registró el peso $\operatorname{seco}(\mathrm{PS}, \mathrm{g})$.

Con los datos de las rodajas se calculó GE = PS/VV, cuyo valor promedio fue de $0.37 \mathrm{~g} \mathrm{~cm}^{-3}$. Este valor se utilizó para transformar el volumen de tallo a biomasa de cada árbol $(\mathrm{BM})$, mediante el producto de volumen verde: $\mathrm{VOL} \times \mathrm{GE}$.

Para estimar biomasa de madera a nivel árbol se ajustaron modelos de regresión, y el mejor ajuste se logró utilizando a DN y ALT de árbol (Rodríguez-Ortiz et al., 2012):

$$
B A M=95.552 \times(\text { DN X ALT })^{-0.5057} \quad \ldots(\text { Ec. } 5)
$$

donde: $\mathrm{BAM}=$ biomasa acumulada en madera $(\mathrm{kg}), \mathrm{DN}=$ diámetro normal $(\mathrm{cm})$, ALT $=$ altura total $(\mathrm{m})$. El ajuste alcanzado fue de $\mathrm{R}^{2}=0.88, \mathrm{CV}=91.3 \%$ y $\mathrm{CME}=134.0, \mathrm{P}<$ 0.0001 .

\section{Estimación de biomasa por componente aéreo en las procedencias y mantillo}

En todos los árboles plantados se midieron las variables involucradas en las Ecs. 3 a 5 (DN, ALT, DCV y LC), para estimar la biomasa acumulada en cada componente aéreo de las procedencias.

En cada unidad experimental se muestreó, aleatoriamente, un rectángulo de $1 \times 0.5 \mathrm{~m}$ de mantillo (piso forestal). Se obtuvo peso fresco (PVM, g) y se seleccionaron al azar tres muestras que se almacenaron en bolsas de papel. Las muestras de mantillo se secaron en estufa a $75^{\circ} \mathrm{C}$ hasta peso constante para determinar su peso seco (PSM, g). El factor promedio obtenido (0.92) de la relación PSM/PVM se utilizó para transformar el peso fresco de mantillo a biomasa, la cual se infirió por hectárea.

El análisis de varianza y pruebas de separación de medias (Tukey, 0.05) y modelos de regresión se solucionaron con el paquete estadístico $\mathrm{r} \AA$.

\section{RESULTADOS Y DISCUSIÓN}

\section{Biomasa en acículas}

La biomasa de acículas mostró variación significativa entre procedencias $(\mathrm{P}<0.0033)$. Los valores más altos fueron para las procedencias Molango y El Piñón, Hidalgo con 5.4 y $5.1 \mathrm{t} \mathrm{ha}^{-1}$, cuya partición de biomasa total a la formación de hojas fue de 19.3 y $17.2 \%$, respectivamente. Sin embargo, la procedencia El Madroño asignó la proporción más alta a este rubro (20.2\%). La biomasa más baja en este componente fue para la procedencia Los Lirios, Coahuila (1.9 t ha ${ }^{-1}$ ), la cual destinó solamente $7.5 \%$ de la biomasa aérea total a la formación de acículas (Cuadros 1 y 2).

La biomasa foliar mostró alta variabilidad entre procedencias $(\mathrm{CV}=62.5 \%)$; la procedencia de mayor variabilidad fue Molango, Hidalgo (desviación estándar, DE = 3.1), y la de menor variación fue la de Los Lirios, Coah. (DE = 1.7).

Los valores más altos de biomasa foliar correspondieron a las procedencias del centro del país con un promedio de $4.44 \mathrm{t} \mathrm{ha}^{-1}$ y $19.2 \%$ de la biomasa total, mientras que los valores más bajos se presentaron en procedencias del norte con promedio de $2.74 \mathrm{t} \mathrm{ha}^{-1}$ y $10.9 \%$ de biomasa total. Al respecto, López-Upton et al. (2004) y Velasco-Velasco et al. (2012) también afirmaron que existen respuestas distintas entre procedencias del centro y norte del país, con base en las características morfológicas de las poblaciones naturales. 
Durante un ciclo de crecimiento el árbol destina recursos a la formación de madera en el fuste solo hasta que se han satisfecho las demandas de producción de yemas, sustancias de reserva y almacenaje (Oliver y Larson, 1996). Por ello, la producción de biomasa foliar se incrementa al final de la fase de establecimiento, cuando se destina mayor cantidad de recurso fotosintético para la producción de hojas que para formar tallos y raíces (Guevara y Guenni, 2004).

La alta variabilidad detectada en la biomasa foliar de las procedencias aquí evaluadas evidencia las diferencias en las estrategias de crecimiento entre las procedencias norteñas y las del centro de México. Además, la proporción de biomasa en la copa disminuye con la edad, contrario a lo que sucede con el fuste (Ferrere et al., 2008). A nivel árbol, las procedencias del centro destinaron una mayor proporción de carbohidratos a la formación de hojas, sobre todo cuando los individuos se liberaron de la competencia, la cual ocurrió por muerte natural (autoaclareo) (Rodríguez-Ortiz et al., 2011 a y b).

\section{Biomasa en ramas}

La biomasa contenida en ramas no mostró diferencias estadísticas $(\mathrm{P}=0.07)$ entre procedencias, a pesar que los valores más altos en biomasa acumulada en ramas correspondieron a El Piñón (13.8 tha ${ }^{-1}$ y Molango (13.1 t ha $\left.{ }^{-1}\right)$, ambas del Estado de Hidalgo, y que el valor más bajo fue para Los Lirios, Coah. (5.2 $\mathrm{t} \mathrm{ha}^{-1}$ ) (Cuadro 1). El problema podría ser originado por las altas varianzas ya que en ramas se ha encontrado el CV más alto (82.8\%) de toda la biomasa aérea (Montgomery et al., 2005).

Las procedencias que destinan mayor cantidad de la biomasa total aérea a ramas fueron del centro del país: Molango (46.9 \%), El Piñón (46.1 \%) y Comunidad Durango Zimapán, todas del Estado de Hidalgo. Por otro lado, los árboles de la procedencia norteña Los Lirios, Coah. sólo destinaron $19.44 \%$ de la biomasa aérea total para la formación de ramas (Cuadro 2).

Las procedencias del centro del país acumularon en promedio $10.40 \mathrm{tha}^{-1}$ en biomasa de ramas, y las procedencias del norte $6.96 \mathrm{t} \mathrm{ha}^{-1}$. La partición de biomasa es una condición intrínseca de cada procedencia, además de que tal partición está asociada con la edad fisiológica de la planta y la condición edafoclimática (García et al., 2009; Medina et al., 2011). Según estudios de Paz y Páez (2002) en Barleria lupulina Lindl., el estrés hídrico afecta la distribución de biomasa. Algo similar podría haber sucedido con las procedencias de Pinus greggii establecidas en el clima seco de la Mixteca, con $650 \mathrm{~mm}$ de precipitación y temperatura de $15{ }^{\circ} \mathrm{C}$.

Cuadro 1. Acumulación de biomasa en acículas, ramas, madera y biomasa total en árboles de 14 años de edad de Pinus greggii procedentes de 13 localidades de México, plantados en Magdalena Zahuatlán, Nochixtlán, Oaxaca.

\begin{tabular}{lcccc}
\hline \multirow{2}{*}{ Procedencias } & \multicolumn{4}{c}{${\text { Biomasa acumulada }\left(\mathrm{t} \mathrm{ha}^{-1}\right)}$} \\
\cline { 2 - 5 } & $5.391 \pm 3.1 \mathrm{a}$ & $13.126 \pm 9.8 \mathrm{a}$ & $9.470 \pm 2.5 \mathrm{~b}$ & $27.987 \pm 12.3 \mathrm{a}$ \\
\hline Molango, Hgo. & $5.161 \pm 2.9 \mathrm{a}$ & $13.805 \pm 10.8 \mathrm{a}$ & $11.01 \pm 5.9 \mathrm{ab}$ & $29.976 \pm 12.7 \mathrm{a}$ \\
El Piñón, Hgo. & $4.769 \pm 3.0 \mathrm{ab}$ & $11.238 \pm 9.2 \mathrm{a}$ & $9.474 \pm 5.3 \mathrm{~b}$ & $25.481 \pm 13.0 \mathrm{a}$ \\
Comunidad Durango, Zimapán, Hgo. & $4.542 \pm 3.0 \mathrm{ab}$ & $9.130 \pm 7.4 \mathrm{a}$ & $8.774 \pm 4.8 \mathrm{~b}$ & $22.446 \pm 11.7 \mathrm{a}$ \\
El Madroño, Qro. & $4.062 \pm 1.5 \mathrm{ab}$ & $9.269 \pm 3.9 \mathrm{a}$ & $8.848 \pm 2.9 \mathrm{~b}$ & $22.179 \pm 6.5 \mathrm{a}$ \\
Laguna Atezca, Hgo. & $3.790 \pm 2.5 \mathrm{ab}$ & $9.187 \pm 9.4 \mathrm{a}$ & $15.343 \pm 11.2 \mathrm{ab}$ & $28.320 \pm 9.9 \mathrm{a}$ \\
Tres Lagunas, Qro. & $3.549 \pm 2.5 \mathrm{ab}$ & $5.820 \pm 4.1 \mathrm{a}$ & $10.103 \pm 5.0 \mathrm{ab}$ & $19.472 \pm 8.4 \mathrm{a}$ \\
Santa Anita, Coah. & $3.377 \pm 2.6 \mathrm{ab}$ & $7.097 \pm 5.8 \mathrm{a}$ & $10.391 \pm 5.2 \mathrm{ab}$ & $20.865 \pm 9.0 \mathrm{a}$ \\
Xochicoatlán, Hgo. & $3.019 \pm 2.2 \mathrm{ab}$ & $6.403 \pm 4.5 \mathrm{a}$ & $15.928 \pm 5.2 \mathrm{ab}$ & $25.350 \pm 8.0 \mathrm{a}$ \\
Puerto San Juan. Coah. & $2.779 \pm 2.1 \mathrm{ab}$ & $6.850 \pm 3.7 \mathrm{a}$ & $14.707 \pm 7.7 \mathrm{ab}$ & $24.336 \pm 7.1 \mathrm{a}$ \\
Jamé, Coah. & $2.714 \pm 2.4 \mathrm{ab}$ & $6.811 \pm 4.8 \mathrm{a}$ & $17.058 \pm 7.6 \mathrm{a}$ & $26.583 \pm 8.8 \mathrm{a}$ \\
Ejido 18 de Marzo, Galeana, N.L & $2.440 \pm 1.8 \mathrm{ab}$ & $10.17 \pm 12.3 \mathrm{a}$ & $15.238 \pm 7.8 \mathrm{ab}$ & $27.848 \pm 12.5 \mathrm{a}$ \\
Puerto Conejos, Coah. & $1.995 \pm 1.7 \mathrm{~b}$ & $5.139 \pm 3.8 \mathrm{a}$ & $19.370 \pm 13.5 \mathrm{a}$ & $26.504 \pm 11.7 \mathrm{a}$ \\
Los Lirios, Coah. &
\end{tabular}

Medias con letras iguales en columnas no son estadísticamente diferentes (Tukey, 0.05). Medias \pm desviación estándar. "Incluye la corteza del fuste. 
Cuadro 2. Partición de biomasa en el componente aéreo y biomasa en mantillo en una plantación de Pinus greggii con árboles de 14 años de edad procedentes de 13 localidades de México, en Magdalena Zahuatlán, Oaxaca.

\begin{tabular}{lcccc}
\hline \multirow{2}{*}{ Procedencias } & \multirow{2}{*}{ Biomasa en mantillo $\left(\mathrm{t} \mathrm{ha}^{-1}\right)$} & \multicolumn{3}{c}{ Partición de biomasa (\%) } \\
\cline { 3 - 5 } & & Acículas & Ramas & Madera con corteza \\
\hline Puerto Conejos, Coah. & $11.20 \pm 6.3 \mathrm{a}$ & 8.8 & 36.5 & 54.7 \\
Santa Anita, Coah. & $11.33 \pm 10.8 \mathrm{a}$ & 18.2 & 29.9 & 51.9 \\
Pto. San Juan. Coah. & $10.92 \pm 8.6 \mathrm{a}$ & 11.9 & 25.3 & 62.8 \\
Los Lirios, Coah. & $9.94 \pm 10.3 \mathrm{a}$ & 7.5 & 19.4 & 73.1 \\
Jamé, Coah. & $11.46 \pm 11.0 \mathrm{a}$ & 11.4 & 28.1 & 60.4 \\
Ejido 18 de Marzo, Galeana, N.L. & $9.56 \pm 8.5 \mathrm{a}$ & 10.2 & 25.6 & 64.2 \\
El Madroño, Qro. & $15.73 \pm 13.4 \mathrm{a}$ & 20.2 & 40.7 & 39.1 \\
Tres Lagunas, Qro. & $12.75 \pm 9.5 \mathrm{a}$ & 13.4 & 32.4 & 54.2 \\
El Piñón, Hgo. & $9.59 \pm 9.7 \mathrm{a}$ & 17.2 & 46.1 & 36.7 \\
Laguna Atezca, Hgo. & $11.18 \pm 12.8 \mathrm{a}$ & 18.3 & 41.8 & 39.9 \\
Molango, Hgo. & $15.49 \pm 12.0 \mathrm{a}$ & 19.3 & 46.9 & 33.9 \\
Xochicoatlán, Hgo. & $10.35 \pm 7.3 \mathrm{a}$ & 16.2 & 34.0 & 49.8 \\
C. Dgo. Zimapán, Hgo. & $13.02 \pm 12.9 \mathrm{a}$ & 18.7 & 44.1 & 37.2 \\
\hline
\end{tabular}

Medias de biomasa en mantillo con letras iguales no son estadísticamente diferentes (Tukey, 0.05). Medias \pm desviación estándar. ${ }^{\dagger}$ Porcentajes con respecto a la biomasa total aérea por árbol.

\section{Biomasa en madera con corteza}

La biomasa en madera con corteza fue diferente estadísticamente $(\mathrm{P}<0.005)$ entre las procedencias de mayor $\mathrm{y}$ menor acumulación, con un $\mathrm{CV}$ de $56.1 \%$. Los valores más altos correspondieron a las procedencias del norte del país: Los Lirios y Ejido 18 de Marzo, con 19.370 y 17.058 t ha $^{-1}$, respectivamente, que fueron superiores a los registrados en las procedencias del centro de México: Laguna Atezca, El Madroño, Molango y Comunidad Durango, cuya biomasa varió entre 8.8 y $9.5 \mathrm{t} \mathrm{ha}^{-1}$ (Cuadro 1). En este contexto las procedencias del centro del país acumularon en promedio $8.21 \mathrm{t} \mathrm{ha}^{-1}$ vs. $15.40 \mathrm{t} \mathrm{ha}^{-1}$ en las procedencias del norte. Las mismas procedencias norteñas destinan la mayor proporción de biomasa total aérea a la formación de madera con corteza (entre 64.2 y $73.1 \%$ ), en contraste con la procedencia de Molango que tuvo la menor proporción (33.9 \%) (Cuadro 2).

Varios investigadores (López y Valencia, 2001; Ferrere et al., 2008; Rodríguez-Ortiz et al., 2011 a y b) han afirmado que al aumentar la competencia, es mayor la diferencia en tamaño entre árboles de la misma edad. A ello se atribuye que en el presente estudio las procedencias norteñas hayan tenido mayor proporción de biomasa en el fuste, porque destinaron una menor proporción de carbohidratos a la formación de hojas.

Esto permite inferir que en las condiciones de la Mixteca oaxaqueña (sur de México), las procedencias de $P$. greggii del norte, dadas las condiciones ambientales y edáficas del lugar de plantación, hayan mostrado mayor eficiencia de crecimiento maderable al destinar mayor prioridad a la acumulación de madera con corteza (Cuadro 2). Estos resultados son coincidentes con Velasco-Velasco et al. (2012), quienes en la misma plantación encontraron en 2004 claras diferencias en crecimiento entre procedencias del norte y centro de México. La densidad de la madera necesariamente está ligada a la proporción de biomasa acumulada en el fuste, y con la edad del árbol y otros factores ambas características van incrementando su variabilidad (López y Valencia, 2001).

\section{Biomasa aérea total y en el mantillo}

La biomasa aérea total entre procedencias no fue estadísticamente diferente $(\mathrm{P}=0.32)$, aunque fue el componente de menor variabilidad $(\mathrm{CV}=39.3 \%)$. Las estimaciones de biomasa total aérea fueron similares entre procedencias, que variaron en un rango de 19.5 a $29.9 \mathrm{t} \mathrm{ha}^{-1}$ (Cuadro 1). Es conocido que la magnitud de la distribución de biomasa depende de factores ambientales y del genotipo (Ramírez et al., 1997; Olalde et al., 2000); las condiciones de manejo y temporalidad también influyen en la partición de biomasa, sobre todo a nivel de individuo (Chave et al., 2001; Rodríguez-Ortiz et al., 2011a).

Los porcentajes de biomasa destinados a los diferentes órganos aéreos muestran una acumulación mayor en madera (50.6\%), seguido de ramas (34.7\%), y por último 
acículas (14.7\%). Estos resultados son similares a los obtenidos por Montes de Oca-Cano et al. (2009) en árboles de $P$. durangensis de entre 3 y 10 años de edad, quienes determinaron $46.9,35.6$ y $17.3 \%$ para madera, acículas y ramas, respectivamente.

La similitud en la partición de biomasa entre $P$. durangensis y $P$. greggii tal vez se deba a la semejanza de edades, ya que la plantación de $P$. greggii tenía 14 años al efectuar este estudio (2011). Al respecto, Montes de Oca-Cano et al. (2009) afirman que en el intervalo de edades de 3 a12 años, la biomasa en madera en $P$. durangensis aumenta $2.96 \%$, en hojas disminuye $3.13 \%$, y en ramas aumenta $0.17 \%$, todos a escalas anuales.

Por su parte, en Abies religiosa Avendaño et al. (2009) reportaron una partición de 84.5, 6.9 y $8.6 \%$ para fuste, ramas y follaje, respectivamente. Además de la especie, la partición de biomasa entre los diferentes órganos de los árboles depende de factores ambientales como el clima, que influye en los fotoasimilados de reserva que contribuyen al rendimiento de las plantas, y como el $\mathrm{pH}$ ya que la acidez del suelo es un factor que disminuye la productividad de la plantación (García et al., 2009).

Los análisis estadísticos no mostraron diferencias significativas $(\mathrm{P}=0.59)$, pero sí alta variabilidad $(\mathrm{CV}=64.1 \%)$ en la biomasa en el mantillo (Cuadro 2). Tales valores fluctuaron de $15.73 \mathrm{tha}^{-1}$ para los árboles del Madroño hasta 9.56 t ha ${ }^{-1}$ para los de la procedencia Ejido 18 de Marzo. Según Rodríguez-Laguna et al. (2009), esta procedencia presenta ventajas para proteger el suelo en el norte del país.

En su estudio realizado en Colombia, Ramírez et al. (2007) reportaron un ingreso de biomasa al piso forestal de $8.36 \mathrm{t} \mathrm{ha}^{-1} \mathrm{año}^{-1}$ en $P$. patula, de $7.87 \mathrm{t} \mathrm{ha}^{-1} \mathrm{año}^{-1}$ en Quercus humboldtii, y de $3.72 \mathrm{t} \mathrm{ha}^{-1}$ año $^{-1}$ en Cupressus lusitanica, todos en bosques naturales. En este contexto, P. greggii tiene un desempeño limitado, ya que en 14 años de la plantación y las condiciones ambientales y climáticas de la Mixteca Oaxaqueña presentó una acumulación en el mantillo de apenas $0.83 \mathrm{t} \mathrm{ha}^{-1} \mathrm{año}^{-1}$, valor poco competitivo con lo que otras especies pueden incorporar anualmente al piso forestal.

\section{CONCLUSIONES}

Las acumulación de biomasa en acículas fue mayor de $5 \mathrm{t}$ $\mathrm{ha}^{-1}$ en las procedencias de Pinus greggii del centro de México (El Piñón y Molango, Hidalgo), en comparación con las procedencias del norte. La biomasa de ramas fue similar entre procedencias, con un promedio de $9.5 \mathrm{t} \mathrm{ha}^{-1}$. La biomasa en madera con corteza de las procedencias del norte del país (Los Lirios, Coahuila y Ejido 18 de Marzo, N.L.) con 19.4 y $17.0 \mathrm{t} \mathrm{ha}^{-1}$ respectivamente, fue superior a la biomasa de este componente en las procedencias del centro de México (Laguna Atezca, El Madroño, Molango y Comunidad Durango) cuya biomasa fluctuó entre 8.8 y $9.5 \mathrm{t} \mathrm{ha}^{-1}$.

La biomasa total aérea fue similar entre procedencias de $P$. greggii, con una acumulación de $25.6 \mathrm{t} \mathrm{ha}^{-1}$. En general, las procedencias del centro de México destinan a la formación de hojas una mayor proporción de la biomasa total aérea, y las procedencias del norte del país destinan más a la formación de madera con corteza. En las condiciones de la Mixteca de Oaxaca, los árboles de P. greggii de 14 años de edad mostraron una partición relativa de biomasa total aérea de $52.0,33.6$ y $14.4 \%$ para madera con corteza, ramas y follaje, respectivamente.

\section{BIBLIOGRAFÍA}

Acosta-Míreles M, J Vargas-Hernández, A Velázquez-Martínez, J D Etchevers-Barra (2002) Estimación de la biomasa aérea mediante el uso de relaciones alométricas en seis especies arbóreas en Oaxaca, México. Agrociencia 60:725-736.

Avendaño H D M, M Acosta M, F Carrillo A, J D Etchevers B (2009) Estimación de biomasa y carbono en un bosque de Abies religiosa. Rev. Fitotec. Mex. 32:233-238.

Bayle R L (1995) Upper-stem volume from stem-analysis data: and overlapping bolt method. Can. J. For. Res. 25:170-173.

Bloomberg M, E G Mason, P Jarvis, R Sedcole (2008) Predicting seedling biomass of radiata pine from allometric variables. New For. 36:103-114.

Chave J, B Riéra, M A Dubois (2001) Estimation of biomass in a neotropical forest of French Guiana: spatial and temporal variability. J. Trop. Ecol. 1:79-96.

Cornejo O E H, E Bucio Z, B Gutiérrez B, S Valencia M, C Flores L (2009) Selección de árboles y conversión de un ensayo de procedencias a un rodal semillero. Rev. Fitotec. Mex. 32: 87-92.

Delgado L, A, M F Acevedo, H Castellanos, H Ramírez, J Serrrano (2005) Relaciones alométricas y patrones de crecimiento para especies de árboles de la reserva forestal Imataca, Venezuela. Interciencia 30:275-283.

Díaz-Franco L, M Acosta-Mireles, F Carrillo-Anzures, E Buendía-Rodríguez, E Flores-Ayala, J D Etchevers-Barra (2007) Determinación de ecuaciones alométricas para estimar biomasa y carbono en Pinus patula Schl. et Cham. Mad. Bosq. 13:25-34.

Domínguez C P A, J J Návar C, J A Loera O (2001) Comparación del rendimiento de pinos en la reforestación de sitios marginales en Nuevo León. Agrociencia 7:27-35.

Ferrere P, A M Lupi, R Boca, V Nakama, A Alfieri (2008) Biomasa en plantaciones de Eucalyptus viminalis Labill. de la Provincia de Buenos Aires, Argentina. Cien. For. 18:293-307.

García A, M Dorado, I Pérez, R Cun, T López, E Montilla (2009) Acumulación de biomasa seca en diferentes órganos de la planta de arroz en condiciones de déficit hídrico. Agron. Trop. 59:15-24.

Guevara E, O Guenni (2004) Acumulación y distribución de biomasa en Leucaena leucocephala (lam) de Wit., durante la fase de establecimiento. I. Repartición de biomasa. Zoot. Trop. 20:147-156.

Kaonga M L, T P Bayliss-Smith (2009) Carbon pools in tree biomass and the soil in improved fallows in eastern Zambia. Agrofor. Syst. 76:37-51.

López L, L, S Valencia M (2001) Variación de la densidad relativa de la madera de Pinus greggii Engelm. del norte de México. Mad. Bosq. 7:37-46.

López-Upton, J, C Ramírez-Herrera, O Plascencia-Escalante, J JassoMata (2004) Variación en crecimiento de diferentes poblaciones de las dos variedades de Pinus greggii. Agrociencia 38:457464.

Medina M G, D E García, P Moratinos, L J Cova (2011) Comparación 
de tres leguminosas arbóreas sembradas en sustrato alcalino durante el periodo de aviveramiento. II. Patrón de crecimiento y distribución de la biomasa. Pastos Forr. 34:167-178.

Montes de Oca-Cano E, P García-Ramírez, J A Nájera-Luna, J MéndezGonzález (2009) Ajuste de ecuaciones de biomasa para Pinus durangensis Martínez en la región del el Salto, Durango. Rev. Chapingo S. Cien. For. Amb. 15: 65-71.

Montgomery D C, E A Peck, G G Vining (2005) Introducción al análisis de regresión lineal. Ed. CECSA, México, D.F. 588 p.

Morante C J, J Alba L, L del C Mendizábal H (2005) Estudio de conos, semillas y plántulas de Pinus greggii Engelm. de una población del estado de Veracruz, México. Foresta Ver. 7:23-31.

Návar C J J, N González B, J J Graciano L, V Dale B Parresol (2004) Additive biomass equations for pine species of forest plantations of Durango, Mexico. Mad. Bosq. 10:17-28.

Olalde G V M, P Sánchez G, L Tijerina C, A A Mastache L, E Carreño $\mathbf{R}$ (2000) Crecimiento y distribución de biomasa en girasol en función del nitrógeno y densidad de población en clima cálido. Terra Latinoam. 18:313-323.

Oliver C D, B C Larson (1996) Forest Stand Dynamics. Wiley. New York, USA. 520 p.

Paz V, A V A Páez (2002) Distribución de biomasa de Barleria lupulina Lindl. en respuesta a tres regímenes de riego. Rev. Fac. Agron. LUZ 20:273-281.

Pimienta de la T, D J, G Domínguez C, O Aguirre C, F Javier H, J Jiménez $\mathbf{P}$ (2007) Estimación de biomasa y contenido de carbono en Pinus coopperi Blanco, en Pueblo Nuevo, Durango. Mad. Bosq. 13:35-46.

Ramírez C J A, C M Zapata D, J D León P, M I González H (2007) Caída de hojarasca y retorno de nutrientes en bosques montanos andinos de Piedras Blancas, Antioquia, Colombia. Interciencia 32:303-311.
Ramírez H C, J J Vargas H, J Jasso M, G Carrillo C, H Guillén A (1997) Variación isoenzimática de diez poblaciones naturales de Pinus greggii Engelm. Agrociencia 31:223-230.

Rodríguez-Laguna R, J Meza-Rangel, J Vargas-Hernández, J JiménezPérez (2009) Variación en la cobertura de suelo en un ensayo de procedencias de Pinus greggii Engelm. en el Cerro El Potosí, Galeana, Nuevo León. Mad. Bosq. 15:47-59.

Rodríguez-Ortiz G, A Aldrete, V A González-Hernández, H M De Los Santos-Posadas, A Gómez-Guerrero, A M Fierros-González (2011a) ¿Afectan los aclareos la acumulación de biomasa aérea en una plantación de Pinus patula? Agrociencia 45:719-732.

Rodríguez-Ortiz G, H M De Los Santos-Posadas, V A González-Hernández, A Aldrete, A Gómez-Guerrero, A M Fierros-González (2012) Modelos de biomasa aérea y foliar en una plantación de pino de rápido crecimiento en Oaxaca. Mad. Bosq. 18:25-41.

Rodríguez-Ortiz G, V A González-Hernández, A Aldrete, H M De Los Santos-Posadas, A Gómez-Guerrero, A M Fierros-González (2011b) Modelos para estimar crecimiento y eficiencia de crecimiento en plantaciones de Pinus patula en respuesta al aclareo. Rev. Fitotec. Mex. 34:205-212.

Rojo-Martínez G E, J Jasso-Mata, A Velázquez-Martínez (2003) Las masas forestales como sumideros de $\mathrm{CO}_{2}$ ante un cambio climático global. Rev. Chapingo S. Cien. For. Amb. 9:57-67.

Valencia M S, M V Velasco G, M Gómez C, M Ruiz M, M A Capó A (2006) Ensayo de procedencias de Pinus greggi Engelm. en dos localidades de la Mixteca Alta de Oaxaca, México. Rev. Fitotec. Mex. 29:27-32.

Velasco-Velasco, V A, J R Enríquez-del Valle, G Rodríguez-Ortiz, G V Campos-Ángeles, M Gómez-Cárdenas, M L García-García (2012) Evaluación de procedencias de Pinus greggii Engelm. en dos plantaciones en la Mixteca Oaxaqueña. Rev. Mex. Cien. For. 3:41-50. 